\title{
Entre topías, utopías y heterotopías. Notas acerca del lugar y las modalidades de la práctica docente en contextos de formación
}

\author{
Topias, utopias and heterotopias. Notes on places and \\ modalities of teaching practice within the training environment
}

Paula Cristina RIPAMONTI ${ }^{1}$

\begin{abstract}
Resumen
El artículo ofrece una reflexión acerca del lugar y las modalidades de la práctica docente en contextos de formación. La intención es articular notas de índole crítica que puedan abrirnos a otras acciones en los trayectos de formación de formadores, desde un pensamiento situado y singular, sin pretensión de generalización y atendiendo al ejercicio docente como una praxis de recomienzos. A partir de un texto de Foucault que caracteriza las heterotopías como contraespacios, reales y posibles, el trabajo analiza las narrativas como modalidad de formación que incluye otras textualidades así como lugares compartidos de aprendizaje (comunidades de indagación).
\end{abstract}

Palabras clave: Práctica docente. Heterotopías. Narrativas. Comunidad de indagación.

\begin{abstract}
This article proposes to analyze the place and modalities of teaching practice within the training environment. The aim is to integrate critical notes that can lead us to perform other actions in the teaching training journey from a situated and singular thought, without generalizing and taking the act of teaching as a restart activity. Based on a Foucault text that characterizes heterotopias as the other space, actual and possible, this job studies narratives as a training modality that includes other textualities as well as shared learning places (communities of inquiry).
\end{abstract}

Keywords: Teaching Practice. Heterotopias. Narratives. Community of Inquiry.

1 Profesora en Filosofía (FFyL, UNCuyo), Especialista en Docencia Universitaria, con Postítulo en Investigación Educativa conenfoque socio-antropológico (CEA, UNCórdoba y MECyT de la Nación) y Doctoranda en Filosofía (FFyL, UNCuyo). Actualmentese desempeńa como Docente de la cátedra Antropología Filosófica (FFyL, UNCuyo) y de Introducción a la Antropología yProblemática del conocimiento (Escuela Normal Superior 9-001, IES). Secretaria Académica de la Dirección de EducaciónSuperior de la Dirección General de Escuelas, Gobierno de Mendoza (período 2003-2007). E-mail: paularip@speedy.com.ar

\begin{tabular}{l|l|l|l|l|l|} 
R. Educ. Públ. & Cuiabá & v. 28 & n. 68 & p. $507-520$ & maio/ago. 2019 \\
\hline
\end{tabular}




\section{Un "entre" como punto de partida}

El objetivo de la presente reflexión es pensar ciertos sentidos del lugar y las modalidades de la práctica docente en contextos de formación. La intención es articular algunas notas de índole crítica que puedan abrirnos a otras acciones y textualidades en los trayectos de formación de formadores, desde un pensamiento situado y singular sin pretensión de generalización y atendiendo al ejercicio docente como una praxis de recomienzos.

La educación en sentido amplio, es decir el campo constituido tanto por instituciones, discursos, prácticas como sujetos, etc., nada entre dos orillas. Una que la ubica dentro del sistema socio-político-cultural vigente y pone la mirada en su función reproductiva o normalizadora y otra, que la concibe como antidestino, como lugar de la igualdad, como posibilidad de cambio o transformación y la dota de una finalidad liberadora, emancipadora es decir, de una función utópica. Ambas miradas poseen una vasta literatura de desarrollo, discusiones e interpelaciones.

En el marco de los recorridos curriculares de las carreras docentes ${ }^{2}$, las denominadas prácticas constituyen un campo complejo, piedra de toque de las trayectorias teóricas previstas y reales como de la pretendida calidad de la formación de las/os futuras/os docentes para desempeñarse profesionalmente en el sistema educativo. Por esto, su presencia, lugar, sentido y aporte a la formación ha

2 En Argentina el sistema de formación docente es dual, es decir, está a cargo de diferentes instituciones: universidades e institutos de educación superior. Y si bien poseen elementos comunes como el hecho mismo de formar y otorgar títulos con competencia para el ejercicio de la docencia en el sistema educativo (esto en el marco de la Ley Nacional de Educación $\mathrm{N}^{\circ} 26.206$ ), también poseen aspectos diferenciales. Uno de ellos tiene que ver con las políticas educativas. Las universidades son autónomas y poseen espacios propios de elaboración de orientaciones pedagógico-curriculares y consensos. Por su parte, los institutos dependen de las provincias y de los marcos de referencia avalados por el Consejo Federal de Educación, constituido por las/os ministras/as de educación del país. En términos de organización y gestión curricular de cada institución, las diferencias se extienden y las brechas existentes en aspectos referidos a financiamiento, condiciones materiales y estructurales de funcionamiento del sistema formador son importantes. La formación docente se realiza a través de carreras de grado (universidades) o lo que se denomina formación inicial (institutos superiores) y se complementa y continúa a lo largo del ejercicio de la profesión a través de posgrados (especializaciones, maestrías, doctorados), postítulos, cursos de desarrollo profesional continuo y certificaciones para quienes no poseen titulación docente. En el país hay 1344 institutos con carreras docentes de los cuales el 58\% son de gestión estatal. En el ańo 2017, cuentan con 604.003 estudiantes ( $80 \%$ asiste a instituciones estatales), de los cuales el $75 \%$ lo constituyen mujeres y hubo 51030 egresados de grado y 40179 en posgrados y postítulos (según Anuario estadístico 2017). En cuanto a las Universidades nacionales más del $50 \%$ forman docentes (según datos del Consejo Interuniversitario Nacional -CIN), de acuerdo con ANFHE, 33 lo hacen en las disciplinas sociales y humanísticas (http://www.anfhe.org.ar/paginas/institucional/integrantes.html). 
convocado muchas de las discusiones y reformas normativas y curriculares de los últimos años ${ }^{3}$. El Campo de la Práctica es un espacio de frontera y de circulación, es el "lugar" constituido por varios lugares, mixtura entre institución formadora y escuelas del sistema educativo donde se realizan experiencias formativas, escuelas a las que denominamos asociadas (o escuelas destino) y que se instituyen como parte del proceso educativo, y por esto, son co-formadoras.

Es en esos espacios de las prácticas donde es posible visibilizar la suerte de batalla irresoluble en la que el sistema justifica su existencia a la vez que advierte de su potencial reproductor y busca afirmarse en su rol en la mejora de la sociedad en la que se inscribe, entre otros resultados. Esto aparece fuertemente y de modo explícito en los trayectos formativos, en especial en los espacios que deben mixturar la transmisión de teorías pedagógicas, estrategias didácticas de enseñanza-aprendizaje con su planificación en vistas a la gestión de intervenciones áulicas. Generalmente, estas prácticas se organizan bajo el mandato de ajustarse a las formas instituidas de enseñar en las escuelas pero con el afán de garantizar propuestas innovadoras, creativas, exitosas.

No son pocas las tensiones existentes entre la enciclopedia ofrecida desde los programas de los diferentes cursos o módulos, que hablan de cambios de paradigmas, de transformación, de ejercicio crítico y hasta de emancipación y liberación y las unidades curriculares que tienen a su cargo acompañar las primeras experiencias docentes, orientar el ingreso a las instituciones y definir lenguajes y formas de planificación de las mismas. En estas últimas, una suerte de colonización de la praxis termina organizando las prácticas de modo orgánico con lo previsto por el sistema, normativa y consuetudinariamente, en un claro ejercicio de "habitus", como lo podríamos describir siguiendo a Bourdieu".

En contextos críticos y ante sucesos sociales y políticos que nos desplazan de la monotonía de la vida cotidiana, por ejemplo, desde hechos criminales aberrantes, discriminaciones de índole social, económica o sexista a discusiones legislativas para instituir nuevos derechos, se alzan voces que reclaman a la educación si no la solución, al menos la tarea de prevención o evitación de situaciones de violencia,

3 En Argentina la Formación docente, tanto en Universidades como Institutos superiores, se organiza curricularmente en cuatro Campos de Formación y uno de ellos es el de las Prácticas Profesionales, los otros corresponden a: Formación General, Formación Disciplinar y Formación Pedagógica. En Institutos, el Campo de la Práctica debe tener el $18 \%$ de la carga horaria total, mientras que en Universidades, la norma prevé un mínimo del 14\% (Res. 1166/2016-CIN).

4 Lo refiero en el sentido de apropiación de esquemas de acción a través de los cuales se reproducen prácticas adaptadas a diversas situaciones. El "habitus" involucra un aprendizaje práctico a través del cuerpo, experiencias, modos de percepción del mundo socialmente estructuradas y estructurantes (Bourdieu, 1980). 
de problemas socio-culturales variados. Es allí donde al ejercicio docente se le impone un accionar que debe proporcionar una educación para el orden pero con y a través de la posibilidad de transformarlo en uno mejor.

Quienes trabajamos en el campo de la formación docente, no desconocemos estas tensiones y mandatos. Tampoco estamos ajenas a ciertas preguntas de figura y fondo como estas: ¿Qué podemos en los recorridos de la formación de formadores? ¿Cuáles son nuestros límites autoimpuestos? ¿Cuáles son nuestros ajustados condicionamientos? ¿Cuáles nuestras aperturas? ¿Qué tanto conocemos, y estamos dispuestas, a hacernos cargo de la heterogeneidad de las aulas y de quienes las habitan? ¿Qué lugar ocupan en nuestras propuestas de formación las historias de vida, las experiencias docentes, los problemas concretos, las operaciones de racialización, las complicaciones afectivas, las dificultades de enseñar y aprender en contextos diversos y más aún en los vulnerables,...? ¿Cuáles modalidades de prácticas estamos pensando para intervenir los mandatos y sus sistemas de clasificación heredados como las polarizaciones (teoría-práctica, rico-pobre, mujer-hombre, blanco-negro, desarrollo-subdesarrollo, saber-ignorancia...) y los criterios de legitimación excluyentes de otras cosmovisiones, lenguas y saberes no occidentales?

Creo que el problema a veces es no tanto evitar las preguntas como el afán por responderlas de forma apresurada y soslayando conflictos. Estamos llenos de pre-comprensiones a-críticas, escasamente interrogadas e ideas románticas sobre la profesión, escasamente realizables.

\section{Un texto para pensar}

En esa tarea y preocupación y desde las experiencias mismas es que construyo algunos mapas de lecturas. A veces resulta determinante dar con una clave para ingresar a un campo y poder movernos en él tanto como interpelarlo. En esta búsqueda di hace poco con un texto de Michel Foucault, "Utopías y heterotopías" $(1966)^{5}$ en el que arriesga a decir que no hay grupo humano que no delimite en el espacio que ocupa y vive, "lugares utópicos" y "momentos ucrónicos". El llama la atención sobre los espacios (en) que las culturas (se) construyen y sus variaciones. En un sentido, esos espacios están constituidos por los tópos concretos o fácticos

5 "Utopías y heterotopías" junto con "El cuerpo utópico" fueron dos conferencias radiofónicas que Foucault dio el 7 y 21 de diciembre de 1966, respectivamente. El tema de la primera aparece nuevamente en otro texto de 1967, "De los espacios otros" y también en el reconocido libro Las palabras y las cosas de 1966. 
que habitamos y clasificamos de muchas formas, como territorios, sitios, regiones (habitación, casa, jardín, calle, ciudad, campo, poblado,...). Estos lugares no constituyen zonas o sitios cerrados, sino que son porosos, ahuecados, penetrables, con relieves y pliegues, niveles y desniveles, etc., y desde ellos siempre proyectamos de algún modo, nos desplazamos, nos movemos.

Un caso lo constituyen las utopías, que "son los emplazamientos sin lugar real" y "mantienen con el espacio real de la sociedad una relación general de analogía directa o inversa", pueden constituirse en la versión perfeccionada o involucionada del tópos presente. Para Foucault, en todos los casos, utopías nostálgicas o proyectivas superadoras del orden vigente o restauradoras, aunque localizadas, siempre son espacios irreales que consuelan (FOUCAULT, 1984, 1985).

Otros lugares lo constituyen una suerte de "contraespacios", "lugares reales fuera de todo lugar", "absolutamente otros" y son las heterotopías (FOUCAULT, 2008, p. 3-4). A lo largo de la historia, en cada sociedad se han inventado estas heterotopías o "utopías realizadas" en el sentido de constituir emplazamientos que inquietan, cuestionan, invierten, subvierten, impugnan las topias en las que vivimos. Poseen formas variadas y no permanecen constantes en el tiempo, desaparecen o devienen en otras. El carácter varía, pueden constituirse como lugares privilegiados, sagrados, prohibidos, ociosos, y los ejemplos van desde el teatro, los cementerios, museos o bibliotecas hasta las clínicas psiquiátricas, los asilos, los burdeles, las sociedades coloniales o las prisiones.

En términos espaciales, la heterotopía yuxtapone "en un lugar real varios espacios que normalmente serían o deberían ser incompatibles" (p. 6) y en este sentido, el teatro es un caso específico o los jardines orientales en los que cabían todas las regiones del mundo. Desde la perspectiva del tiempo y como si "pudiera estar fuera de tiempo" (p. 7), la heterotopía lo corta o detiene, extiende o eterniza, como un museo, o singulariza y se vuelve una crónica, como una fiesta. En otros casos, una heterotopía puede estar ligada al tiempo de los pasajes y las transformaciones, como los colegios. Finalmente, para Foucault, las heterotopías "tienen siempre un sistema de apertura y cierre" en relación con los espacios que las rodean. Las hay cerradas, abiertas, penetrables las que parecen abiertas pero exigen iniciación (p. 8), como sitios para visitantes de paso o de prácticas rituales.

Si las utopías consuelan, las heterotopías inquietan impugnando lo real. Y lo hacen al crear una ilusión, que denuncia a lo real como ilusión o disipa lo real desde la fuerza del juego, el sueño, la aventura. Por esto los niños habitan heterotopías que inventan y en un jardín, una cama o una caja pueden volar, nadar, esconderse, volverse invisibles, convertirse en fantasmas, etc. 
En Las palabras y las cosas, podemos leer otro rasgo de las heterotopías y es el de intervenir el lenguaje, no sólo rompiendo los modos tradicionales en que nombramos las cosas y arruinando la sintaxis con la que las juzgamos, sino desafiando aquel "secreto" "menos evidente que hace 'mantenerse juntas' (unas al otro lado o frente de otras) a las palabras y a las cosas" (1985, 3). Las utopías entonces "permiten las fábulas y los discursos: se encuentran en el filo recto del lenguaje" mientras que las heterotopias "detienen las palabras en sí mismas y desafían, desde su raíz, toda posibilidad de gramática; desatan los mitos y envuelven en esterilidad el lirismo de las frases (1985, 3).

Esos otros lugares (heterotopías) coexistiendo en estos lugares reales (topías) yuxtaponen lo posible a la vez que nos sustraen los emplazamientos en los que nos sostenemos, el terreno firme no sólo de nuestras verdades sino de las matrices o estructuras o esquemas a través de los cuales podemos comprender una u otra cosa/ nombre del mundo. En los términos del Prefacio, se nos sustrae la "mesa de disección" (3) como marco dentro del cual algo adquiere sentido, algo que agrupamos, organizamos, ponemos en relación, designamos. En el cruce lenguaje y espacio, las heterotopías también invitan a tomar un riesgo, el de que se puede "estar" de otro modo y "hacer" otras cosas con otras o, quizás, las mismas, palabras.

\section{Algunas notas acerca del lugar y la modalidad de las prácticas}

Los procesos de profesionalización del ejercicio docente tuvieron como dispositivo vital la formalización de los lenguajes con los que nos referimos a nuestras prácticas concretas. Fue así tanto que el acrecentamiento de horas de prácticas en las carreras docentes como su presencia desde el primer ańo de la formación estuvo fuertemente motivada por aumento del acervo de estos lenguajes en los que había que formar, así teorías del aprendizaje y del desarrollo como objetivos, expectativas de logro, habilidades, capacidades, competencias, proyectos, planificaciones, secuencias didácticas, etc. necesitaban mucha atención.

Sin embargo, las prácticas docentes desde la perspectiva de la modalidad de formación continuaron siendo un núcleo difícil de intervenir y subvertir. Me refiero a pensar las prácticas como un tipo de saber específico, práctico, permeada por discusiones teóricas que desmantelen la colonización de una racionalidad instrumental, que afirmen su carácter político y condición ideológica. Esto exige impugnar la dicotomía moderna de saber experto (teoría)/ saber falso o pseudo saber (experiencia), disputar otras formas de legitimidad y discutir el criterio de 
verdad como adecuatio ${ }^{6}$ (que determina que una vez obtenida la verdad, se aplica técnicamente, sin más, al campo de la experiencia). Es necesario visibilizar el carácter cultural, heterogéneo y complejo, constitutivo de la vida en las aulas porque es el de la vida humana misma también.

En esta línea, resulta pertinente incorporar dos notas críticas que Andrea Alliaud apunta a los procesos de enseñanza y transmisión. Una tiene que ver con el supuesto de que las respuestas para solucionar problemas de la práctica están siempre "afuera" de ella (en cursos, capacitaciones, posgrados...), otra es que en esta dinámica receptiva, el sujeto de "intervención" es siempre individual (ALLIAUD, 2011, p. 62). Y yo agregaría, que ese sujeto casi siempre es nombrado con género masculino, representa la "cultura" y los saberes básicos, prioritarios, mínimos, objetivos, y no sólo recibe desde "afuera" las respuestas sino que él mismo está afuera de las etnias, las comunidades culturales, los contextos críticos, vulnerables, socialmente fragmentados, racializados, estigmatizados, está afuera de los contextos y los problemas mismos. Es un estar fuera que lo deja extranjero en su propio quehacer y unilateraliza su práctica.

La lectura de Foucault me resultó una provocación para proponer las prácticas docentes como heterotopías. Estoy pensando en interpelar ese otro estar afuera extranjero al que me referí. Así las cosas, abordar las prácticas desde una perspectiva crítica-genealógica nos sitúa en otro mapa de la cuestión. En primer lugar, no desconocer que se trata de un acto político, es decir, que se juega en relaciones vitales de mutua afectación, de forma situada y singular, por eso acontece siempre en un lugar y puede fundar experiencias, como también puede producir su propio olvido. Se trata de un campo (un sembradío podría ser la imagen) de experiencias que producimos y nos producen, nos configuran, nos hacen. Como tal, podemos reinventarlas como heterotopías, esos otros lugares posibles y presentes, antes que como utopías, que remiten a lo que aún no tiene lugar y desplaza el tiempo de realización a un futuro que no siempre podrá reparar, efectuar, realizar el derecho a la educación.

Localizadas, yuxtapuestas, diferentes, porosas, atravesadas por el tiempo al modo de corte o extensión, las heterotopías (escolares, pedagógicas, educativas, comunitarias,...) son los contraespacios que tienen efectuación, que están en los espacios instituidos resistiéndolos. No en vistas a la consecución utópica de

6 Me refiero con esta concepción de verdad a aquella que la entiende como correspondencia con los hechos. Desde esta perspectiva, una afirmación o un enunciado es verdadero en la medida que se adecua a la realidad (la expresa o la representa cabalmente), así la verdad es ajuste o coincidencia entre pensamiento y realidad. 
un objetivo postergado (por irrealizable en el presente) sino en vistas al instante "ahora y aquí" de articulación de un derecho concreto de aprender a leer una lengua (mi lengua, otras lenguas), de viajar por las regiones del mundo, de soñar con un cuento, de resolver un problema de matemática, de evitar una pelea dialogando, de cuidar nuestros cuerpos o elegir la sexualidad, de narrar lo que nos pasa, de escuchar y ser escuchadas/os.

Aceptada esta mirada, las modalidades de las prácticas en contextos de formación docente pueden articularse en otras textualidades, precisamente aquellas capaces de hacerse cargo de los problemas, los sentidos y su articulación crítica en recorridos situados y considerando sin excepción a los incidentes críticos como claves en los procesos de formación (SARDI, 2013). Modalidades que abandonen las predicciones por anticipaciones de sentidos, desplacen los lenguajes representativos y universales y se orienten a través de tramas argumentativas que cuenten y hablen de esos lugares en los que aconteció un encuentro, con sus tensiones y conflictos y sus derivas de resolución o no resolución, en un tiempo propio articulado por el ritmo de las intervenciones.

Las narrativas pueden constituirse en una modalidad de las prácticas y una forma de articular el campo específico en las trayectorias de formación docente. Entiendo por narrativa un modo discursivo, un texto que articula una experiencia, la expresa, la interviene, la inscribe, la constituye, la transmite, desde una trama en la que se implican sujetxs, miradas, significados, situaciones vitales, contextos en un mundo complejo, atravesado por alguna tensión o conflicto. La narración es "una forma artesanal de la comunicación. No se propone transmitir el puro 'en sî́ del asunto, como una información o un reporte" (BENJAMIN, 2008, p. 71).

En sentido amplio, podemos entender la narrativa como un ejercicio, como un texto que escribimos, que leemos, que hablamos y a través del cual realizamos una operación de salvación, nombramos nuestras acciones, las objetivamos, las constituimos en experiencias, las (des)armamos, re-configurándolas a través de su problematización y exposición, de los sentidos que exploramos y buscamos comprender. Otro aspecto vinculado a este ejercicio tiene que ver con la relación entre vida humana y temporalidad. Desde la filosofía, este tema ha recibido múltiples tratamientos y no pretendo aquí desplegarlos. Me interesa señalar, desde nuestra condición de seres históricos y la densidad de ese tiempo que llamamos presente, que narrando(nos) la operación de salvación también lo es de recolección y proyección, de recuperación y anticipación, de (re)construcción de la memoria. Como sostiene Jorge Larrosa, la experiencia "es aquello que nos pasa" como acontecimiento y por esto, "presupone... una articulación temporal" (LARROSA, 2003, p. 614). 
Se trata de la textualidad de una experiencia (SARDI, 2013) que la expone desde sus problemas e incidentes críticos, que nos exige revisar los modos de intervenirlos, resolverlos, trocarlos en saberes comunicados, no como procedimientos formales, sino como cualidades de un quehacer posible en un determinado contexto. Un narrativa se configura desde lo que los lenguajes formalizados encubren, callan, no dicen, explicitando las condiciones materiales, los contextos (social, político, económico, cultural), la presencia de las/os diferentes actoras/es, las pre-comprensiones, las relaciones de poder, las tramas de resolución o no resolución (RIPAMONTI, 2017).

Narrar las prácticas deviene así un dispositivo que permite transformar la realidad de las prácticas e imaginar otros modos de intervenir didácticamente, de aprender, de enseñar contemplando la complejidad de los contextos contemporáneos. Es un modo de legar, también, a las generaciones en formación y a las futuras la heterogeneidad de nuestras prácticas como experiencia acumulada para enfrentar los desafíos de la práctica y para comprender con más claridad las transformaciones pasadas, presentes y futuras (SARDI, 2017, p.13).

Hablar, contar y escribir son momentos del narrar. Pero la narrativa como modalidad de formación no debería ser un recurso aislado o una simple estrategia de evaluación, tampoco un trabajo individual, aislado o extranjero en el sentido de una producción endogámica diferencial. Exige un lugar de apertura, cruce y relaciones, exige rehacer y deshacer colectivamente prácticas que suelen sacralizar y normalizar acciones como si fueran meras técnicas pedagógicas a aplicar. Por eso la narrativa como modalidad requiere contar los "secretos" de los encuentros, de esas relaciones que, invisibles, niegan la performatividad de nuestros lenguajes.

El pedagogo argentino Daniel Suárez ha desarrollado una metodología que propone la documentación narrativa de experiencias como una modalidad de trabajo pedagógico entre docentes a través de talleres en los que estos escriben, reescriben, leen, conversan, promoviendo la participación de los mismos en procesos de indagación, desarrollo profesional y acción, desde relaciones de saber y poder horizontales (SUÁREZ, 2016). Si bien esta propuesta está orientada al trabajo con docentes en ejercicio, hay elementos que resultan interesantes para incluir en la modalidad narrativa de la etapa de la formación inicial a la que me refiero. Entre ellos, los espacios donde se provocan encuentros de escritura, escucha y reescritura en los términos de una conversación pedagógica con fuerte sentido epistémico.

En los contextos de formación es fundamental constituir estas instancias que pueden asumir diferentes características: ateneos, talleres, colectivos y redes, comunidades de indagación, diálogo o cuestionamiento. Me interesa aquí referirme brevemente a estas últimas, es decir, a la constitución de comunidades de indagación como clave de trabajo en esta modalidad de formación. 


\section{Narrativas como modalidad de formación: textualidades / espacios / instancias}

\author{
Escrituras \\ Biográfica \\ Biográfica escolar \\ Guión conjetural \\ Experiencias \\ pedagógicas
}

\author{
Oralidades \\ Comunidades de \\ indagación/ diálogo/ \\ cuestionamiento \\ Ateneos \\ Talleres de escritura \\ Colectivos y redes \\ narrativas
}

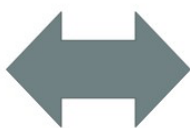

En el campo de la Filosofía con Niños y Jóvenes, las comunidades de indagación son conocidas porque constituyen un dispositivo de trabajo fundamental, si se quiere una metodología de quehacer filosófico y poseen, podríamos afirmar, una especificidad e historia propia ${ }^{7}$. Desde este campo, tomo algunas ideas y prácticas que pueden aportar a la formación docente en el sentido que planteo en este trabajo.

7 Se menciona a Charles Peirce como mentor de la noción “comunidades de investigación” y la idea de que se hace investigación en la medida en que se parte de una duda que busca ser respondida (y fijada en una creencia) para luego volver a ser sometida a duda; de este modo el ejercicio de cuestionar resulta vital y esto en el marco de un proceso colectivo de trabajo en una comunidad que va fijando y discutiendo criterios de verdad. En los ańos 60 y en el contexto de un modelo pragmatista, Mathew Lipman, un educador preocupado por los altos índices de fracaso académico en jóvenes universitarios, retomó la idea de la educación como proceso social y la importancia de aprender a través de comunidades de investigación. Lipman creó un programa (con una metodología y materiales específicos) y determinó cuáles prácticas debían ser promovidas en estas comunidades para alcanzar aprendizajes vinculados al pensamiento lógico y reflexivo. Un aspecto fundamental para Lipman es incluir la dimensión afectiva y siguiendo a John Dewey, provocar y problematizar la "experiencia" educativa a través de discusiones filosóficas colectivas. Un texto clásico y de lectura obligatoria relativo a las comunidades de indagación es el de Laurance Splitter y Ann Sharp, La otra educación. Filosofía para niños y la comunidad de indagación (Buenos Aires, Manantial, 1996). Para un panorama del estado de discusión en torno a este campo cfr. KOHAN, W. y WAKSMAN, V. (ed.), Filosofía para niños. Discusiones y propuestas y KOHAN, W. Teoría y práctica en filosofía con niños y jóvenes. Experimentar el pensar, pensar la experiencia. 
Las comunidades de indagación en el contexto de la formación pueden constituirse en comunidades de diálogo (como encuentros de co-formación). Estos espacios, entre otros aspectos, tienen que garantizar (inventar) "heterotópicamente" (si se me permite el término):

- Una disposición circular de los cuerpos (con posibilidad de las miradas)

- La transversalidad de la palabra (otras legitimidades, otras subjetividades)

- La horizontalidad de las relaciones (en términos de potencias que se cruzan y no relaciones jerárquicas de poder)

- La autonomía de su práctica (autodeterminando las condiciones del diálogo y criterios de legitimidad)

- Una concepción del saber en términos de construcción y como práctica de (re)comienzos

- El trabajo a través de agenda de preguntas, es decir, a partir de los problemas que propone (construye, formula, visibiliza, nombra) la propia comunidad

Lo que acontece en el contexto de las comunidades se orienta a concebir una praxis de los (re)comienzos, en la que importan los sentidos de la presencia (¿quiénes somos? ¿por qué estamos aquí?), lo que hacemos y lo que nos pasa con lo que hacemos (nuestras experiencias). Resulta vital en una comunidad de co-formación nombrar los problemas, trabajarlos desde el hacernos cargo colectivamente. Se trata de un lugar otro donde se trabaja con agenda de preguntas y no con un plan de tarea. Estas comunidades producen discursos y pueden ejercer la fuerza del juego, los sueños, las aventuras. Como dice Leonor Arfuch (a propósito de un trabajo sobre memoria e historia reciente), "la palabra intrínsecamente ligada a la configuración de la experiencia, la idea de comunidad que sostiene el más simple intercambio de las voces y aún, de su huella en la escritura. Una palabra, en este caso, cuya insistencia se opone al vacío, a la ausencia, al olvido. La palabra -la narración-como acto de resistencia” (ARFUCH, 2010, 40).

Propongo, entonces, el lugar de las prácticas como heterotopías y una modalidad narrativa que incluyen otras textualidades como una forma de aprender a hacer en contraespacios constituidos colectivamente. "Las narrativas, más que imponer, suscitan cursos de acción, abren interrogantes y promueven respuestas diversas. Proponen, provocan, intrigan, sorprenden y dejan pensando..." (ALLIAUD, 2011, 89).

Mi experiencia docente en formación de formadores, tanto en institutos como universidad, también ha navegado entre esas dos orillas que mencioné al comienzo. A través de diferentes investigaciones educativas, busqué marcos 
conceptuales y analíticos para comprender lo que hacemos cuando decimos que formamos docentes. En ese camino cada vez más me encuentro casi desarrollando esa disciplina que pensó Foucault como "heterotopología”, "cuyo objeto serían esos espacios diferentes" (1966, p. 4), cada vez más me descubro trabajando en comunidades de indagación, con agenda de problemas y en círculos mirando(nos) y atravesada por los saberes que producimos desde las narraciones de experiencias.

Para terminar entonces quiero dejar abierto lo dicho aquí con el relato de una profesora de Filosofía, en un "contraespacio" de un taller que tuve el gusto de coordinar (en la Especialización en Filosofía con Niños y Jóvenes) y en el que su autora nos contó de sus encuentros con chicos de secundaria, los modos en que se sintió ante sus expresiones y cómo comenzó a (re)construir su propuesta pedagógica. Gabriela tituló su narrativa "Mayéutica trágica para un tiempo liminar" y eligió comenzar citando expresiones de sus estudiantes:

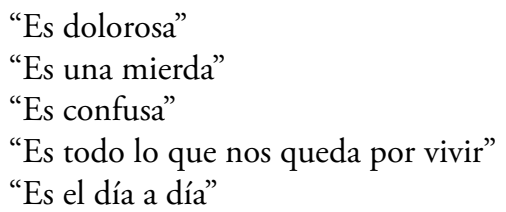

Estas son respuestas a la pregunta, intempestiva quizá, ¿qué es la realidad? que formulé a los 20 estudiantes de $5^{\circ} 1^{\text {a }}$ de la escuela secundaria donde trabajo. Tal pregunta tenía un objetivo: conocer qué pensaban sobre ese concepto y, esperaba que me respondieran con alguna referencia a una objetividad, a un mundo exterior opuesto al mundo interior...

Los autores de estas respuestas son chicos y chicas caracterizados por colegas como "apáticos", "desmotivados", "especiales", "sin iniciativa”, "descomprometidos", "imposibles de mover".

En esa ocasión pude registrar cuidadosamente cada una de las respuestas, las intervenciones que siguieron y los momentos de diálogo que hubo entre algunos estudiantes; me sorprendió que el diálogo no brotara a borbotones, por lo que intervine con nuevas preguntas, tratando de indagar qué sentidos encerraba la palabra realidad para los estudiantes. En un momento, les hice una observación acerca de sus respuestas, mostrando el fuerte aspecto individual y subjetivo, hasta "psicológico" de ellas, por lo que me mira L. V. y dice ¿"cuál es la diferencia entre filosofía y psicología"? Hacia el final de ese diálogo, dice C. F. "Hay un olor a depresión acá, guacho. Me cambió el ánimo" y todos rieron y coincidieron.

Miré a N. M. y tenía una expresión de incomodidad. Al finalizar la clase le pregunté qué había cambiado en él después de la "charla" con sus compañeros y me dijo: "No me gusta que hablen de depresión, yo tengo depresión". 
Estos alumnos provienen, de distintos departamentos del Gran Mendoza, con realidades socio-económicas algo difíciles, cuyas trayectorias escolares no han sido continuas en la mayoría de los casos. Con incertidumbre acerca de qué sea de ellos al finalizar la secundaria. Aunque algunos saben que quieren continuar con estudios superiores y qué van a estudiar, muchos otros no lo saben aún. Vislumbran la dificultad de insertarse en el mundo laboral y "hacerse de una vida" a su medida.

Frente a estas miradas acerca de la realidad, basándome en el trato cotidiano con ellos, me encuentro ante una situación que puedo llamar de desesperanza, de sentimiento de impotencia, de certeza del dolor. En cierta forma, de falta de sentido de la propia vida. En jóvenes de 18 ańos aproximadamente.

No puedo dejar de pensar que la Filosofía tiene algo que ofrecer, hay algo que hacer con la Filosofía. Con urgencia. No puedo pretender que no veo estas respuestas como problemáticas e incluso, inadmisibles en la escuela pública. No porque desee censurar dichas respuestas ni porque eso "no pueda decirse en la escuela", sino porque tiendo a pensar que esa desesperanza podría ahogar posibilidades e iniciativas en personas tan jóvenes, que están terminando su educación secundaria. Porque pensaba que los niños o jóvenes albergan la curiosidad, el asombro y que siendo parte de una comunidad de chicos que se conocen desde hace tiempo y han compartido una historia juntos, podrían también compartir "[...] el goce de conocer, el goce de pensar juntos y de explorar los inacabables caminos de sentido, descubierto y creado en ese medio nutriente que es el diálogo" (SANTIAGO, 2002, p. 8). Y veo que no es siempre así. (SARAVIA, Gabriela, 2018)

\section{Referências}

ALLIAUD, A. Narraciones, experiencia y formación. In ALLIAUD, A.; SUAREZ, D. 3/ El saber de la experiencia. Narrativa, investigación y formación docente. Buenos Aires: CLACSO Ediciones, 2011.

ARFUCH, L. Sujetos y narrativas. En Acta Sociológica, N ${ }^{\circ}$ 53, septiembrediciembre de 2010, pp. 19-41.

BOURDIEU, P. El sentido práctico. Madrid: Taurus, 1980.

BOURDIEU, P.; J.-C. PASSERON. La reproducción. Elementos para una teoría del sistema de enseńanza. Barcelona: Laia, 1972.

FOUCAULT, M. Topologías (1966). En Fractal, N48, enero-marzo, 2008, Año XII, Vol. XII. 
FOUCAULT, M. De los espacios otros "Des espaces autres". Conferencia dicada en el Cercle des études architecturals (14 de marzo de 1967). En Architecture, Mouvement, Continuité, $N^{\circ} 5$, octubre de 1984. Traducida por Pablo Blitstein y Tadeo Lima.

FOUCAULT, M. Las palabras y las cosas. Buenos Aires: Planeta-Agostini, 1985.

KOHAN, W. y WAKSMAN, V. (ed.), Filosofía para nińos. Discusiones y propuestas. Buenos Aires: Novedades educativas, 2004.

KOHAN, W. (comp.). Teoría y práctica en filosofía con nińos y jóvenes. Experimentar el pensar, pensar la experiencia. Buenos Aires: Novedades educativas, 2006.

SARAVIA, Gabriela. "Mayéutica trágica para un tiempo liminar”. Narrativa del Taller de Diseño de Proyectos- Especialización en Filosofía con Niños y Jóvenes. Mendoza, Mimeo, 2018.

RIPAMONTI, P. Investigar a través de narrativas. Notas epistémicometodológicas. En ALVARADO, M.; DE OTO, A. Metodologías en contexto. Buenos Aires: Clacso, 2017.

SARDI, V. La escritura de las prácticas en la formación docente en Letras. En SARDI, V. Relatos inesperados. La escritura de incidentes críticos en la formación docente en Letras. La Plata: Edulp, 2013.

SARDI, V. Escribir la práctica, inscribir la experiencia. En Saberes y prácticas. Revista de Filosofía y Educación. Año 2, N² 2, 2017.

SPLITTER; L., SHARP, A. La otra educación. Filosofía para nińos y la comunidad de indagación (Buenos Aires: Manantial, 1996.

SUAREZ, D. Escribir, leer y conversar entre docentes en torno de relatos de experiencia. En Revista Brasileira de Pesquisa (Auto)Biográfica, Salvador, v. 01, n. 03, p. 480-497, set./dez. 2016. 